\title{
Transición del cuidado pediátrico al cuidado adulto ¿una realidad para la cual no estamos preparados?
}

\author{
Transition from pediatric to adult care. Are we prepared for this reality? \\ Ángela Mabel Urrea-Sepulveda1* orcid.org/0000-0001-8244-5481 \\ María Belén Tovar-Añez² orcid.org/0000-0002-6138-9924
}

1 Programa de Especialización en Pediatría. Universidad de La Sabana. Bogotá, Colombia.

2 Posgrados Facultad de Medicina. Universidad de La Sabana. Bogotá, Colombia.

\section{Resumen}

Introducción: La sobrevida de pacientes pediátricos con enfermedades crónicas cada vez es mayor a nivel mundial, tendencia que es similar en Colombia. La mayoría de estos pacientes realizaran la transición a la adultez y con ésta a los servicios médicos de adultos. Objetivo: Evidenciar la importancia de hacer una adecuada transición del paciente con enfermedades crónicas, del cuidado pediátrico a la atención del adulto, describir las características, barreras, riesgos y consecuencias de un proceso inadecuado. Materiales y métodos: Se realizó revisión de la literatura a partir de la búsqueda de información relacionada. Resultados: Se seleccionaron 50 artículos en diferentes revistas académicas. Con la información expuesta se describen las definiciones de enfermedad crónica, transición y transferencia al cuidado adulto, los riesgos de los adolescentes con enfermedades crónicas, la preparación y los diferentes pasos para hacer la transición y las consideraciones especiales. Conclusiones: La literatura a nivel mundial recalca la importancia de la realización de un proceso de transición esencial para garantizar el mantenimiento de la salud en los pacientes con enfermedades crónicas, sin embargo, no se encuentran guías o literatura al respecto realizadas en Colombia.

Palabras clave: Pediatría; transición; adolescentes; enfermedades crónicas; cuidado. (Fuente: DeCS, Bireme).

\begin{abstract}
Introduction: Survival of pediatric patients with chronic diseases is increasing worldwide, a trend that is also seen in Colombia. Most of these patients will make the transition to adulthood and adult medical services. Objective: To analyze the transition process of patients with chronic diseases from pediatric to adult care and describe the characteristics, barriers, risks, and consequences of this process. Materials and methods: A literature review search for related information. Results: 50 articles were selected from different academic journals. The assembled information was used to describe: (i) definitions of chronic disease, (ii) transition and transfer to adult care, (iii) risks of adolescents with chronic diseases, (iv) preparation and the different steps to make the transition, and (v) special considerations. Conclusions: The existing literature highlights the importance of undertaking an essential transition process to ensure the maintenance of health in patients with chronic diseases. However, in Colombia there are no guidelines or literature on the subject.
\end{abstract}

Keywords: Pediatrics; transition; adolescents; chronic diseases; care. (Source: DeCS, Bireme).

\footnotetext{
*Autor de correspondencia

María Belén Tovar-Añez

e-mail: mariatoa@unisabana.edu.co
} 


\section{Introducción}

La incidencia de las enfermedades crónicas en la edad pediátrica ha aumentado en las últimas décadas como consecuencia de la mejoría progresiva a nivel de tecnologías y tratamientos disponibles, lo cual a su vez ha generado un aumento considerable en la expectativa de vida de niños y adolescentes con enfermedades que previamente llevaban a una muerte prematura(1).

La definición de enfermedad crónica en pediatría se establece como: "cualquier condición que conlleve a una deficiencia biológica, psicológica y cognitiva con evolución mayor a un año o que se considere que por sus características será de carácter permanente"(2). Estas condiciones hacen que el niño o adolescente sea más vulnerable, tenga mayores necesidades $\mathrm{y}$ requiera mayor soporte por parte del personal de salud y sus cuidadores(3).

La tendencia mundial es que la mayoría de los niños y adolescentes con enfermedades crónicas llegarán a la adultez y como consecuencia serán transferidos para valoración y seguimiento por un médico internista o especialista en medicina familiar, los cuales están enfocados en el cuidado del paciente adulto(4-6); esto es válido entre otros, para pacientes con cardiopatías congénitas, enfermedades reumatológicas o endocrinológicas crónicas, y prematuros extremos teniendo en cuenta que por la mayor tasa de sobrevida en forma secundaria ha aumentado la incidencia de alteraciones neurocognitivas y necesidades especiales(7-10). En Estados Unidos se calcula que anualmente 750.000 adolescentes realizan la transición del cuidado pediátrico al cuidado adulto y que probablemente esta cifra aumentará con el tiempo(11); en varias cohortes de pacientes con enfermedades crónicas pediátricas se ha observado una mayor tasa de mortalidad entre los adultos jóvenes de 18 a 21 años, en comparación con el grupo de los adolescentes de 13 a 17 años y aquellos pacientes que superan los 21 años(12-14).

En el año 2011 la Academia Americana de Pediatría (AAP), estimó que en Estados Unidos un 10-15\% de la población menor a 18 años presentaba algún tipo de enfermedad crónica, es decir entre 13 a 15 millones de niños y adolescentes tienen alguna de estas condiciones $(1,3,15)$. En la tabla 1 se presenta una relación de algunas condiciones y patologías crónicas observadas en los infantes. En Colombia la incidencia y prevalencia de las enfermedades crónicas no ha sido plenamente establecida, sin embargo, se han publicado estimaciones como en el estudio de Bernal et al.(16), donde estimaron que las enfermedades genéticas en Colombia probablemente tienen una frecuencia que oscila entre 37,3 y 52,8 por cada 1.000 habitantes. En esta cifra se incluyen las malformaciones congénitas que corresponden de manera global al 50\% del total, es decir que para el año 2025 aproximadamente 2,8 millones de colombianos tendrán una enfermedad de origen genético con la consecuente carga económica y pérdida de años de vida útil(16).

Tabla 1. Ejemplos enfermedades crónicas en pacientes pediátricos

\begin{tabular}{l}
\hline \multicolumn{1}{c}{ Enfermedades crónicas en la edad pediátrica } \\
\hline Fibrosis quística \\
Cardiopatías congénitas \\
Asma \\
Disquinesias ciliares \\
Enfermedades neuromusculares (atrofia muscular, distrofias \\
musculares) \\
Parálisis cerebral \\
Epilepsia \\
Diabetes mellitus \\
Espina bífida \\
Síndrome de Down \\
Anemia de células falciformes \\
Trastornos del espectro autista \\
Trastornos de déficit de atención e hiperactividad TDHA \\
Enfermedad intestinal crónica. \\
Obesidad \\
Trastornos de la alimentación. \\
Alteraciones del neurodesarrollo \\
Déficit cognitivo \\
Enfermedades reumatológicas (LES, AIJ) \\
Trasplantados \\
VIH/SIDA \\
Sobrevivientes del cáncer infantil \\
Errores innatos del metabolismo \\
\hline
\end{tabular}

En el marco de la práctica médica colombiana, poco se ha tratado el tema de la transición de niños y adolescentes que padecen enfermedades crónicas y son atendidos en servicios médicos pediátricos, para pasar a servicios médicos de adultos. El objetivo de esta revisión fue evidenciar la importancia de hacer una adecuada transición del paciente con enfermedades crónicas, del cuidado pediátrico a la atención del adulto, describir las características, barreras, riesgos y consecuencias de un proceso inadecuado. La compilación de información disponible hará más eficiente la comprensión del tema para futuros lectores. De igual forma evidenciará las carencias dentro de las pesquisas 
existentes, dando paso a nuevos debates y al interés por seguir investigando.

\section{Materiales y métodos}

Se realizó una revisión teórica de la información relacionada, consultando en las bases Pubmed y Lilacs; por un lado, Pubmed, engloba la mayoría de los trabajos realizados en habla inglesa y por el otro, Lilacs publicaciones producidas desde Latinoamérica, para mostrar cómo se está trabajando el tema en los países cercanos a Colombia, ya que las condiciones de los sistemas de salud y los contextos sociales resultan ser parecidos. Se utilizaron palabras claves en español e inglés como: transición/transition, adolescente/teenager, preparación/readiness. Como criterios de inclusión se consideraron únicamente artículos completos que hayan sido publicados en los últimos 10 años y presenten los resultados de pacientes entre los 0 a los 18 años.

\section{Resultados}

Inicialmente Se obtuvo 65 artículos que cumplían con los criterios de inclusión. Después de esta primera etapa de selección, se hizo uso de las referencias existentes en los mismos, dando paso a la revisión de otras investigaciones que también fueron incluidas. Para la revisión final se incluyeron 50 artículos en idioma inglés y español a partir del año 2010 y algunos anteriores como referente histórico, Teniendo en cuenta la exploración realizada, se encontró que hay varios factores y actores para tener en cuenta dentro del proceso de transición del cuidado pediátrico al cuidado adulto en el marco de enfermedades crónicas. Por ello se agrupan y se describen las características de los actores durante el proceso, los riesgos del paciente y las intervenciones necesarias para lograr una adecuada culminación, que permita un mejor desarrollo y calidad de vida de los pacientes adolescentes y adultos jóvenes, cuestión que es expuesta a lo largo de este documento.

\section{¿Transferencia o transición?}

Existen diferencias importantes entre los conceptos de transferencia y transición(6). La transferencia es el proceso pasivo de cambiar de servicio del paciente, es decir que el paciente al cumplir la mayoría de edad deja de asistir a los servicios médicos pediátricos y asiste a los servicios médicos de adultos. En contraposición la transición se considera un proceso activo a realizarse durante las etapas de maduración del adolescente, cuyo objetivo es asegurar la continuidad del adecuado cuidado, integrando al paciente al sistema de atención del adulto $(6,17)$. En el proceso de transición es imperativo involucrar el equipo médico (pediátrico y de adultos), así como el paciente y sus cuidadores ${ }^{(14)}$. Aun cuando es claro que la transferencia debe ser realizada, no obstante, en nuestro modelo de salud ocurre de manera sistemática al cumplir 18 años, sin importar que se haya o no realizado un proceso de transición, generando dificultades en la atención y consecuencias no deseadas $(6,14,17)$.

La Academia Americana de Pediatría (AAP), la Academia Americana de Médicos Familiares y la Academia Americana de Médicos Internistas han enfatizado en conjunto la necesidad de contar con un plan de transición para garantizar el adecuado acceso a los servicios de salud y adherencia al tratamiento de una manera ininterrumpida durante el proceso de crecimiento y maduración(14). En este mismo documento se reconoce que existen muchas dificultades en el proceso.

\section{Riesgos del adolescente con enfermedad crónica}

La adolescencia es un periodo de profundos cambios cognitivos, emocionales y corporales; en esta etapa del desarrollo psicosocial el adolescente desafía a sus padres o cuidadores, para tener autonomía y crear su propio sistema de valores; estas reacciones propias del adolescente hacen parte del desarrollo normal y están asociadas a la culminación del desarrollo cerebral encaminado a formar un ser autónomo y funcional en la sociedad(18). Estos cambios maduracionales inciden directamente en la forma de pensar y actuar del adolescente, en las relaciones cambiantes con sus pares y padres, impactando de manera importante en el continuo de salud y enfermedad, por lo tanto, estas diferencias evolutivas deben ser consideradas durante el proceso de transición.

Adicionalmente a los cambios emocionales propios de la edad, el adolescente con enfermedad crónica se enfrenta a desafíos de índole emocional mayores a sus pares; al estrés asociado al diagnóstico, a la necesidad de asistir a constantes citas médicas y recibir múltiples tratamientos, se suman las posibles dificultades para relacionarse con sus pares y la marginalización que puedan sufrir en diferentes ámbitos sociales(17). Los adolescentes con enfermedades crónicas tienen un mayor riesgo de ideaciones e intentos suicidas comparados con adolescentes sanos(19-21). Durante el periodo de la 
adolescencia se inicia y consolida el plan de vida, sin embargo, la incertidumbre y expectativas generadas por la enfermedad y su pronóstico son especialmente estresantes para el adolescente, pudiendo dificultar su desarrollo personal $(22,23)$.

El adolescente con enfermedad crónica también se ve expuesto a conductas de riesgo: en este sentido, la pobre adherencia al tratamiento y la negativa a seguir indicaciones médicas es reportada de manera habitual por los médicos y los padres; estas dificultades podrían considerarse en consecuencia a la oposición propia de la edad a los mandatos paternos(17). Igualmente se ven expuestos a otro tipo de conductas de riesgo propias de la edad tales como consumo de sustancias legales e ilegales y las relacionadas con actividades sexuales, cuya prevalencia puede ser similar a la de aquellos adolescentes sin enfermedades crónicas(24).

El agravante es que muchas de estas conductas pueden deteriorar la condición clínica basal, por ejemplo, las enfermedades respiratorias como el asma o la fibrosis quística podrían empeorar con el tabaquismo, igualmente la asociación entre tabaquismo $\mathrm{y}$ diabetes $\mathrm{o}$ del tabaquismo $\mathrm{y}$ enfermedades reumatológicas con el aumento de riesgo cardiovascular y empeoran en algunas condiciones fisiológicas como el embarazo(25-28).

\section{Preparación para la transición}

\section{Preparación del adolescente y de su núcleo} familiar: Los padres o cuidadores están acostumbrados a ser los principales responsables en el cumplimiento de los tratamientos, adicionalmente toman la mayoría de las decisiones relacionadas con los cuidados de sus hijos adolescentes, minimizándoles la capacidad de generar responsabilidad con respecto a sí mismos y a su enfermedad(29). Esto crea dificultades durante la adolescencia, ya que el paciente desafía a sus padres incumpliendo o negándose a recibir los tratamientos prescritos, como forma de dar a entender que también puede decidir. Igualmente, para los padres es difícil aceptar que el adolescente pronto será un adulto en la potestad de decidir los tratamientos a recibir y el principal responsable de su cuidado(29,30).

Las familias o cuidadores de niños con enfermedades crónicas deben tener en cuenta y tratar con los especialistas a cargo y con el sistema de salud, el plan de transición a una edad temprana de la enfermedad (algunos autores recomiendan hacerlo desde el diagnóstico de la enfermedad). A pesar de que los padres o cuidadores son los responsables legales del niño hasta la mayoría de edad, se debe alentar al niño en un proceso de asentimiento e inclusión en las decisiones que le conciernen a su salud y su vida desde temprana edad, y se le debe proporcionar información adecuada (según su condición cognitiva), para que comprenda y se involucre en el manejo de su enfermedad(4, 25, 26, 29, 31-33).

El nivel educativo de los padres genera un impacto directamente proporcional en este aspecto, ya que los padres con mayor nivel educativo serán más capaces de comprender y lograr transferir el conocimiento a sus hijos(34). Así como se presentan dificultades en la transición en el núcleo de apoyo primario, también se presentan en el equipo médico, como se expone más adelante.

Preparación del equipo médico: Gran parte de las dificultades se originan en las diferencias entre el cuidado pediátrico y la atención dirigida al paciente adulto(9). La atención pediátrica de manera tradicional se enfoca principalmente en la supervisión del crecimiento y desarrollo, con una perspectiva de manejo a corto plazo, delegando la responsabilidad (asistencia a citas, administración de medicamentos, etc.) y toma de decisiones a los cuidadores. Generalmente se producen importantes vínculos emocionales entre el pediatra y la familia( ${ }^{(9)}$. En contraste, la atención enfocada en adultos maneja una perspectiva a largo plazo, delegando la responsabilidad y decisiones casi de manera exclusiva al paciente teniendo poco en cuenta a la familia(9).

La necesidad de realizar el proceso de transición y no una transferencia, es la dificultad que presentan los pacientes (adolescentes y adultos jóvenes) para adaptarse a un nuevo modelo de atención, sobre todo en cuanto a los cambios en los roles a asumir, la relación con sus nuevos médicos tratantes y los sentimientos con respecto al anterior personal de salud; estas condiciones impactan directamente en la morbilidad y bienestar del paciente(35-37).

Las recomendaciones internacionales indican que para realizar una adecuada transición ambos equipos médicos deben coordinarse y cumplir unas tareas específicas para hacer el proceso lo más adecuado posible, a pesar de esto aún existen dificultades en esta coordinación y no se ha comprobado que alguna intervención sea superior, por lo que se requiere 
mayor investigación al respecto(38-41), puesto que todas las intervenciones coinciden en la importancia de transferir conocimiento y empoderar al paciente acerca de su enfermedad y los cuidados que requiere, en un marco que permita su desarrollo integral(16).

El médico o equipo pediátrico involucrados en la atención integral del niño deben entender que el paciente crecerá y deberá continuar con el equipo de atención de adultos, y debe ser informado desde el inicio de la relación médico-paciente(29). Es deber del pediatra proporcionar educación clara al paciente fomentando su independencia, dependiendo del momento del desarrollo en el cual se encuentre. Debe cerciorarse que el paciente entienda su enfermedad y sea capaz de relatar su historia clínica, además debe modular los lazos afectivos que se han creado durante la etapa de crecimiento del adolescente, para hacer la transición menos traumática(42). Adicionalmente se ha identificado la necesidad de incluir en los currículos de los programas de pediatría del país educación formal en estos aspectos(43).

Los padres y pacientes perciben que su médico pediatra tiene una mayor experticia y al tener una relación más cercana y afable les es difícil romper los lazos afectivos, los cuales son una barrera importante en el momento de transición. Es importante que el pediatra guíe a los padres durante este proceso para que logren entender que los cambios de roles de paternidad hacen parte de la transición, ya que los padres presentan importante ansiedad durante el proceso y expresan sensación de abandono e inseguridad $(42,44)$.

Por último, el pediatra debería tener la posibilidad de comunicarse con el servicio receptor, lo cual es especialmente difícil en nuestro medio dado que es el asegurador quien lo asigna. El servicio receptor de adultos debería contar con la información del paciente previo al ingreso de este y poder comentar con el pediatra acerca del plan de manejo y ajustes. Es importante recalcar que una de las múltiples barreras para la transición, es el desconocimiento por parte de estos profesionales en el manejo de enfermedades originadas en el periodo pediátrico, sobre todo aquellas de origen genético. Dada esa falta de información, muchos pacientes a pesar de su edad continúan asistiendo a servicios pediátricos de hospitalización y consulta externa $(4,5,45)$.

Los pacientes usualmente describen a los internistas como distantes, poco preocupados por su enfermedad, aunque muchos reportan que sienten mayor libertad de actuación respecto al servicio pediátrico. Por otra parte, los padres reportan sentirse totalmente excluidos e ignorados en el proceso, desconfiando de los conceptos y recomendaciones del médico internista $(37,42)$. Lo ideal es que las partes involucradas, pacientes, familia y personal de salud trabajen de forma conjunta, para obtener una transición planificada y lo menos traumática posible, disminuyendo las complicaciones y dificultades descritas.

\section{Proceso de transición}

Múltiples organismos internacionales, entidades académicas y organizaciones gubernamentales han reconocido la importancia de guías que faciliten el proceso de transición. Los estudios a la fecha indican que, aunque hay avances, existen dificultades en el proceso y dadas las disparidades en las poblaciones no hay evidencia de que alguna intervención sea superior a la otra. Las guías coinciden en que debe ser un proceso ordenado enfocado en la educación gradual del paciente y actividades de autocuidado para garantizar el seguimiento en el tiempo y el mantenimiento de la salud(12, 25, 26, 32, 37, 39, 46-48). A continuación, se resumen las más importantes recomendaciones de estas guías.

\section{Componentes de la transición}

1. Planear la transición de forma previa, educando al paciente y la familia o cuidadores. La AAP y otros autores recomiendan iniciarlo desde los catorce años de vida del paciente, incluyendo la preparación del personal de salud a cargo de este periodo, planeación del cuidado hasta los dieciocho años y la preparación del paciente, su familia o cuidadores $(9,12,14,29,46)$.

2. Conformación de equipos de salud emisores y receptores. El equipo emisor debe estar liderado por el pediatra tratante, quién debe dirigir el proceso de cambio, que prevenga y trate las complicaciones que se puedan presentar y proporcione herramientas claras para el autocuidado e independencia del paciente. Así mismo, el equipo receptor debe estar liderado por un internista o especialista en Medicina Familiar, quien debe estar entrenado en atención de jóvenes con necesidades especiales, que pueden ser demandantes en el servicio de salud. El líder del grupo debe generar una relación médico-paciente en la que se tenga en cuenta las opiniones del paciente, se establezca un 
ambiente de confianza que promueva la independencia en el joven y el desarrollo de destrezas para que participe de forma activa en la promoción de la salud y en la prevención de la complicación de la enfermedad(12, 29, 32, 46).

3. Interacción de los equipos. La interacción es fundamental tanto en el equipo emisor, como en el receptor, ya que se genera un escenario en el que el intercambio de información del paciente, experiencia y conocimientos fortalece el bagaje tanto científico como emocional, afectivo y demás en ambos grupos, lo cual influirá en el beneficio del paciente y en el proceso que va a experimentar $(26,29,46)$.

4. Información del paciente. La información debe ser trasmitida de forma clara, precisa, que permita desarrollar estrategias futuras en la atención. Se debe incluir datos anteriores relevantes que hayan motivado cambios en los tratamientos y los resultados de estos, efectos adversos y secundarios, esquemas de vacunación cumplidos, estudios imagenológicos, etc. (12, 26, 29, $32,39,46,48)$.

5. El paciente y la familia o cuidadores. Es fundamental el apoyo del núcleo primario del paciente, especialmente en aquellos jóvenes con discapacidad, para los cuales el proceso de cambio y adaptación es mucho más difícil o incluso improbable $(12,29,32,39,46,47,49)$.

6. El sistema de salud. El sistema de salud debe proveer los recursos suficientes para que se efectúe este proceso, teniendo en cuenta que beneficia al paciente, minimiza el impacto y el desconcierto natural generados por el cambio, favoreciendo la continuidad en la atención ${ }^{26,29) .}$

\section{Consideraciones especiales}

Paciente con enfermedad Neurológica y déficit cognitivo: Adicionalmente a las limitaciones físicas, los pacientes con enfermedad o condiciones neurológicas pueden tener alteraciones cognitivas que limitan la independencia de manera parcial o completa, con alteración importante para el desarrollo social y académico. Incluso hasta el $80 \%$ de los pacientes adultos con diagnóstico de epilepsia de inicio en la infancia, aun con control de la enfermedad, pueden presentar algún tipo de déficit cognitivo, alteración comportamental o comorbilidad psiquiátrica(49). La parálisis cerebral es un claro ejemplo de condición neurológica cuya incidencia se ha mantenido en el tiempo, actualmente la mayoría de los pacientes con parálisis cerebral sobrevivirán a la adultez siendo un importante reto de manejo para sus padres y equipo médico(35). Es importante el reconocimiento de las alteraciones cognitivas durante la infancia para preparar a los padres y definir el requerimiento de procesos de interdicción que den el poder legal a los cuidadores para permitir continuidad en el acceso de los servicios de salud.

Dependiendo de su grado de discapacidad, promover la transición e independencia en los pacientes con alteraciones neurológicas puede ser un reto importante para el pediatra(49). En los pacientes con discapacidad severa, la transición puede ser más difícil dadas las marcadas diferencias del cuidado pediátrico al del adulto; las decisiones, comunicación con personal médico y cuidado será exclusivo por parte de los padres o cuidadores, con las consiguientes dificultades con el médico internista. Igualmente existe falta de conocimiento en el manejo de los pacientes por parte de los médicos de adultos dada la ausencia de enseñanza en los currículos de los

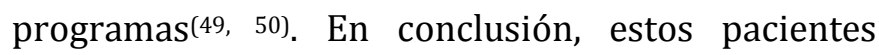
representan un reto mayor para todos los actores en el proceso de transición, requiriendo enfoques diferentes ajustados a cada paciente de acuerdo con las condiciones de desarrollo(50).

\section{Discusión}

La mayoría de los niños y adolescentes con enfermedades crónicas llegarán a la adultez y deben ser transferidos para continuar seguimiento por un médico internista o especialista en medicina familiar, los cuales están enfocados en el cuidado del paciente adulto $(4,5,6)$. En la literatura revisada se documenta la diferencia entre transferencia (proceso pasivo de cambiar de servicio del paciente) y transición, siendo esta ultima el proceso activo a realizarse durante las etapas de maduración del adolescente, con el objetivo de asegurar la continuidad del cuidado, y la integración del paciente crónico con necesidades especiales al sistema de atención del adulto(6,17).

Existen diferencias entre el cuidado pediátrico y el cuidado del adulto: en cuanto al enfoque, el cuidado pediátrico se centra en el crecimiento y el desarrollo, el del adulto en las perspectivas a largo plazo y en evitar las complicaciones; en segundo lugar, la estrategia utilizada en el cuidado pediátrico es el contacto con toda la familia, mientras que en el del adulto la responsabilidad es exclusiva del paciente y la familia en su mayoría no se involucra(9). En el cuidado pediátrico las decisiones las toma la familia con el apoyo de un equipo interdisciplinario, mientras 
que en la atención del adulto las decisiones las toma el paciente y el quipo es multidisciplinario(9).

El proceso de transición requiere una preparación tanto del adolescente como del núcleo familiar, desde el momento del diagnóstico, es decir en etapas tempranas de la enfermedad, ofrecer información veraz y oportuna, e involucrar al paciente en la toma de decisiones $(4,25,26,29-33)$. Por otra parte, debe haber una adecuada coordinación entre los equipos médicos, emisor y receptor; lo ideal es que las partes involucradas, pacientes, familia y personal de salud trabajen de forma conjunta, para obtener una transición planificada y lo menos traumática posible(38-41).

Las guías revisadas coinciden en que la transición debe ser un proceso ordenado enfocado en la educación gradual del paciente y en actividades de autocuidado para garantizar el seguimiento en el tiempo y el mantenimiento de la salud; en líneas generales proponen etapas secuenciales que incluyen una planeación previa enfocada en la educación al paciente y a la familia o cuidadores, conformación e interacción de equipos de salud emisores y receptores, adecuada información y apoyo al paciente y la familia, e integración con el sistema de salud para proveer los recursos suficientes para que se efectúe el

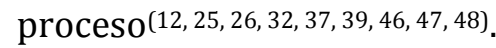

Se hace mención especial a la transición de pacientes con alteraciones neurológicas y déficit cognitivo, requiriendo enfoques diferentes ajustados a cada paciente de acuerdo con las condiciones de desarrollo(50).

\section{Conclusiones}

La prevalencia de las enfermedades crónicas en la edad pediátrica como consecuencia de la mejoría progresiva de tratamientos disponibles ha generado un aumento en la expectativa de vida de niños y adolescentes con enfermedades que previamente llevaban a una muerte prematura. Ante esta situación se hace necesario realizar un adecuado proceso de transición, de la atención pediátrica de estos pacientes hacia servicios de atención de adultos. Este proceso se debe hacer en forma progresiva, involucrando al equipo médico (pediátrico y de adultos), así como el adolescente y su núcleo familiar, puesto que la enfermedad de su hijo, también los afecta en la realización de sus vidas.
El objetivo de la transición es permitir que los pacientes sean lo más independientes posible y tengan la mejor calidad de vida. Aun cuando es claro que la transferencia debe ser realizada, no obstante, en nuestro modelo de salud ocurre de manera sistemática al cumplir 18 años, sin importar que se haya o no realizado un proceso de transición, generando dificultades en la atención y consecuencias no deseadas. El éxito del proceso se evidencia en la capacidad del paciente de tomar sus propias decisiones en salud.

Se resalta la importancia de la comunicación entre los diferentes servicios médicos, en este caso el servicio médico emisor y receptor de pacientes adolescentes para asegurar un adecuado proceso de transición; de igual forma, la responsabilidad que el sistema médico de cada país tiene sobre estos trámites. En los pacientes con discapacidad neurológica o cognitiva se deben tener en cuenta aspectos legales y se debe adaptar el proceso de transición de acuerdo con las capacidades de estos.

No se encontraron estudios realizados a nivel nacional que muestren los procesos de transición del cuidado pediátrico al cuidado adulto. Se hace necesario plantear investigaciones al respecto, incluir en los currículos de posgrado de Pediatría la formación en contenidos que faciliten un adecuado proceso de transición de los adolescentes a los servicios de atención de adultos $\mathrm{y}$, por otra parte, generar guías en el país para ser aplicadas en las diferentes instituciones.

\section{Referencias}

1. Van Dyck PC, Kogan MD, McPherson MG, Weissman GR, Newacheck PW. Prevalence and Characteristics of Children With Special Health Care Needs. Arch Pediatr Adolesc Med [Internet]. el 1 de septiembre de 2004;158(9):884-90. Disponible en: https://doi.org/10.1001/archpedi.158.9.884

2. McPherson M, Arango P, Fox H, Lauver C, McManus M, Newacheck PW, et al. A New Definition of Children With Special Health Care Needs. Pediatrics [Internet]. el 1 de julio de 1998;102(1):137-139. Disponible en: http://pediatrics.aappublications.org/content/102/1/137.a bstract

3. Newacheck PW, Strickland B, Shonkoff JP, Perrin JM, McPherson M, McManus M, et al. An Epidemiologic Profile of Children With Special Health Care Needs. Pediatrics [Internet]. el 1 de julio de 1998;102(1):117-123. Disponible en:

http://pediatrics.aappublications.org/content/102/1/117.a bstract

4. Kovacs AH, McCrindle BW. So hard to say goodbye: transition from paediatric to adult cardiology care. Nat Rev Cardiol [Internet]. 2014;11(1):51-62. Disponible en: 
https://doi.org/10.1038/nrcardio.2013.172

5. Perrin JM, Bloom SR, Gortmaker SL. The Increase of Childhood Chronic Conditions in the United States. JAMA [Internet]. el 27 de junio de 2007;297(24):2755-9. Disponible en: https://doi.org/10.1001/jama.297.24.2755

6. Salmerón Ruiz M., Casas Rivero J, Guerrero Azola F. Problemas de salud en la adolescencia. Patología crónica y transición. Pediatr Integr [Internet]. 2017;XXI(4). Disponible en: https://www.pediatriaintegral.es/publicacion-201706/problemas-de-salud-en-la-adolescencia-patologiacronica-y-transicion/

7. Goodman DM, Hall M, Levin A, Watson RS, Williams RG, Shah SS, et al. Adults With Chronic Health Conditions Originating in Childhood: Inpatient Experience in Children's Hospitals. Pediatrics [Internet]. el 1 de julio de 2011;128(1):5-13. Disponible en: https://pediatrics.aappublications.org/content/128/1/5.sh ort

8. Kapellen TM, Kiess W. Transition of adolescents and young adults with endocrine diseases to adult health care. Best Pract Res Clin Endocrinol Metab [Internet]. 2015;29(3):50513. Disponible

en: http://www.sciencedirect.com/science/article/pii/S15216 90X15000238

9. White PH, Ardoin S. Transitioning Wisely: Improving the Connection From Pediatric to Adult Health Care. Arthritis Rheumatol (Hoboken, NJ). abril de 2016;68(4):789-94. Available: https://doi.org/10.1002/art.39554

10. Racine E, Bell E, Yan A, Andrew G, Bell LE, Clarke M, et al. Ethics challenges of transition from paediatric to adult health care services for young adults with neurodevelopmental disabilities. Paediatr Child Health [Internet]. febrero de 2014;19(2):65-8. Disponible

https://pubmed.ncbi.nlm.nih.gov/24596475

11. McManus MA, Pollack LR, Cooley WC, McAllister JW, Lotstein D, Strickland B, et al. Current status of transition preparation among youth with special needs in the United States. Pediatrics. junio de 2013;131(6):1090-7. DOI: 10.1542/peds.2012-3050

12. Okumura MJ, Kleinhenz ME. Cystic Fibrosis Transitions of Care: Lessons Learned and Future Directions for Cystic Fibrosis. Clin Chest Med [Internet]. 2016;37(1):119-26. Disponible en: http://www.sciencedirect.com/science/article/pii/S02725 2311500146X

13. Wray J, Frigiola A, Bull C. Loss to specialist follow-up in congenital heart disease; out of sight, out of mind. Heart. abril de 2013;99(7):485-90. DOI: 10.1136/heartjnl-2012-302831

14. Hepburn C, Cohen E, Bhawra J, Weiser N. Supporting the Health Care Transition From Adolescence to Adulthood in the Medical Home. Pediatrics [Internet]. el 1 de julio de 2011;128(1):182-200. Disponible en: http://pediatrics.aappublications.org/content/128/1/182.a bstract

15. Davis AM, Brown RF, Taylor JL, Epstein RA, McPheeters ML. Transition Care for Children With Special Health Care Needs. Pediatrics [Internet]. el 1 de noviembre de 2014;134(5):900-908. Disponible en: http://pediatrics.aappublications.org/content/134/5/900.a bstract

16. Bernal Villegas J, Suárez Obando F. La carga de la enfermedad genética en Colombia, 1996-2025 [Internet]. Bogotá: Universitas Médica. 2008; 49 (1):12-28. Disponible en: https://repository.javeriana.edu.co/bitstream/handle/105
54/45685/UniversitasMedica2008v49n1p12-

28.pdf? sequence=1\&isAllowed=y

17. Sawyer SM, Drew S, Yeo MS, Britto MT. Adolescents with a chronic condition: challenges living, challenges treating. Lancet (London, England). abril de 2007;369(9571):1481-9. DOI: 10.1016/S0140-6736(07)60370-5

18. Spear LP. Adolescent Neurodevelopment. J Adolesc Heal [Internet]. 2013;52(2, Supplement 2):S7-13. Disponible en: http://www.sciencedirect.com/science/article/pii/S10541 39X12002078

19. Ferro MA, Rhodes AE, Kimber M, Duncan L, Boyle MH, Georgiades K, et al. Suicidal Behaviour Among Adolescents and Young Adults with Self-Reported Chronic Illness. Can J Psychiatry [Internet]. 2017/08/17. diciembre de 2017;62(12):845-53. Disponible en: https://pubmed.ncbi.nlm.nih.gov/28814100

20. Nock MK, Green JG, Hwang I, McLaughlin KA, Sampson NA, Zaslavsky AM, et al. Prevalence, correlates, and treatment of lifetime suicidal behavior among adolescents: results from the National Comorbidity Survey Replication Adolescent Supplement. JAMA psychiatry [Internet]. marzo de 2013;70(3):300-10. Disponible en: https://pubmed.ncbi.nlm.nih.gov/23303463

21. MacKinnon N, Colman I. Factors Associated with Suicidal Thought and Help-Seeking Behaviour in Transition-Aged Youth versus Adults. Can J Psychiatry [Internet]. 2016/09/24. diciembre de 2016;61(12):789-96. Disponible en: https://pubmed.ncbi.nlm.nih.gov/27578771

22. Maslow GR, Haydon AA, Ford CA, Halpern CT. Young adult outcomes of children growing up with chronic illness: an analysis of the National Longitudinal Study of Adolescent Health. Arch Pediatr Adolesc Med [Internet]. marzo de 2011;165(3):256-61. Disponible en: https://pubmed.ncbi.nlm.nih.gov/21383274

23. Maslow GR, Haydon A, McRee A-L, Ford CA, Halpern CT. Growing up with a chronic illness: social success, educational/vocational distress. J Adolesc Health [Internet]. 2011/02/04. agosto de 2011;49(2):206-12. Disponible en: https://pubmed.ncbi.nlm.nih.gov/21783055

24. Ssewanyana D, Nyongesa MK, van Baar A, Newton CR, Abubakar A. Health risk behavior among chronically ill adolescents: a systematic review of assessment tools. Child Adolesc Psychiatry Ment Health. 2017;11:32. Available: https://doi.org/10.1186/s13034-017-0172-5

25. Foster HE, Minden K, Clemente D, Leon L, McDonagh JE, Kamphuis S, et al. EULAR/PReS standards and recommendations for the transitional care of young people with juvenile-onset rheumatic diseases. Ann Rheum Dis. abril de 2017;76(4):639-46. doi: 10.1136/annrheumdis-2016210112.

26. Buschur EO, Glick B, Kamboj MK. Transition of care for patients with type 1 diabetes mellitus from pediatric to adult health care systems. Transl Pediatr. octubre de 2017;6(4):373-82. DOI: 10.21037/tp.2017.09.06

27. Foster H, Minden K, Clemente D, Leon L, McDonagh J, Kamphuis S. EULAR/PreS standards and recommendations for the transitional care of young people with juvenile-onset rheumatic diseases. Ann Rheum Dis. junio de 2018;77(6):960. doi: 10.1136/annrheumdis-2016-210112

28. Selvaag AM, Aulie HA, Lilleby V, Flatø B. Disease progression into adulthood and predictors of long-term active disease in juvenile idiopathic arthritis. Ann Rheum Dis. enero de 2016;75(1):190-5. DOI: 10.1136/annrheumdis-2014206034 
29. C M, A M, C N, G M, Navarrete C, D M, et al. Transición al cuidado de la vida adulta, de niños y adolescentes con necesidades especiales de atención en salud: recomendaciones del comité NANEAS de la Sociedad Chilena de Pediatría. Rev Chil pediatría. el 1 de junio de 2011;82:238-44.

Available: http://dx.doi.org/10.4067/S0370-41062011000300010

30. Hepburn CM, Cohen E, Bhawra J, Weiser N, Hayeems RZ, Guttmann A. Health system strategies supporting transition to adult care. Arch Dis Child. junio de 2015;100(6):559-64. DOI: $10.1136 /$ archdischild-2014-307320

31. White PH, Cooley WC. Supporting the Health Care Transition From Adolescence to Adulthood in the Medical Home. Pediatrics [Internet]. el 1 de noviembre de 2018;142(5):e20182587. Disponible en: http://pediatrics.aappublications.org/content/142/5/e201 82587.abstract

32. Nicolarsen J. Transition of Adolescents and Young Adults with Congenital Heart Disease: Challenges, Progress, and Future Improvements. Pediatr Ann. el 1 de junio de 2017;46:e224-8. DOI: 10.3928/19382359-20170519-02

33. Mylotte D, Pilote L, Ionescu-Ittu R, Abrahamowicz M, Khairy $\mathrm{P}$, Therrien J, et al. Specialized Adult Congenital Heart Disease Care. Circulation [Internet]. 2014;129(18):1804-12. Disponible https://www.ahajournals.org/doi/10.1161/CIRCULATION AHA.113.005817

34. Javalkar K, Fenton N, Cohen S, Ferris M. Socioecologic Factors as Predictors of Readiness for Self-Management and Transition, Medication Adherence, and Health Care Utilization Among Adolescents and Young Adults With Chronic Kidney Disease. Prev Chronic Dis [Internet]. 2014;11:E117. Disponible http://dx.doi.org/10.5888/pcd11.140072

35. Carroll EM. Health Care Transition Experiences of Young Adults With Cerebral Palsy. J Pediatr Nurs. 2015;30(5):e15764. DOI: $10.1016 /$ j.pedn.2015.05.018

36. Lewis J, Slobodov G. Transition from Pediatric to Adult Health Care in Patients with Chronic Illnesses: An Integrative Review. Urol Nurs [Internet]. 2015;35(5):231-237. Disponible http://europepmc.org/abstract/MED/26630779

37. Inostroza Quezada C, Correa Venegas ML, Besoain Arrau C, Reinoso Medinelli A, Velarde Lizama M, Valenzuela Mujica MT, et al. El proceso de transición de servicios pediátricos a adultos: visión de adolescentes hospitalizados portadores de enfermedades crónicas. Vol. 87, Revista chilena de pediatría. scielocl ; 2016. p. 110-5. Available: http://dx.doi.org/10.1016/j.rchipe.2015.07.014

38. Le Roux E, Mellerio H, Guilmin-Crépon S, Gottot S, Jacquin P, Boulkedid R, et al. Methodology used in comparative studies assessing programmes of transition from paediatrics to adult care programmes: a systematic review. BMJ Open [Internet]. el 1 de enero de 2017;7(1):e012338. Disponible en: http://bmjopen.bmj.com/content/7/1/e012338.abstract

39. Weissberg-Benchell J, Shapiro JB. A Review of Interventions Aimed at Facilitating Successful Transition Planning and Transfer to Adult Care Among Youth with Chronic Illness. Pediatr Ann. mayo de 2017;46(5):e182-7. DOI: 10.3928/19382359-20170421-01

40. Campbell F, Biggs K, Aldiss SK, O'Neill PM, Clowes M, McDonagh J, et al. Transition of care for adolescents from paediatric services to adult health services. Cochrane database Syst Rev. abril de 2016;4:CD009794. doi:
10.1002/14651858.CD009794.pub2.

41. Coyne B, Hallowell SC, Thompson M. Measurable Outcomes After Transfer From Pediatric to Adult Providers in Youth With Chronic Illness. J Adolesc Heal [Internet]. 2016;60(1):3$16 . \quad$ Disponible en: https://www.jahonline.org/article/S1054-139X(16)30177$\mathrm{X} /$ fulltext

42. Van Staa AL, Jedeloo S, van Meeteren J, Latour JM. Crossing the transition chasm: experiences and recommendations for improving transitional care of young adults, parents and providers. Child Care Health Dev. noviembre de 2011;37(6):821-32. DOI: 10.1111/j.13652214.2011.01261.x

43. Flores SP, Tovar MB, Leon MX, Villegas K, Villamizar D del pilar, Granados CE. Caracterización del conocimiento en cuidado paliativo pediátrico y percepción debarreras por parte de los pediatras y residentes de pediatría. Med Paleativa [Internet]. 2015;22(4):127-35. Disponible en: https://www.elsevier.es/en-revista-medicina-paliativa337-articulo-caracterizacion-del-conocimiento-cuidadopaliativo-S1134248X14000469

44. Hergenroeder AC, Wiemann CM, Cohen MB. Current Issues in Transitioning from Pediatric to Adult-Based Care for Youth with Chronic Health Care Needs. J Pediatr. diciembre de 2015;167(6):1196-201. DOI: 10.1016/j.jpeds.2015.08.005

45. Cruikshank M, Foster HE, Stewart J, Davidson JE, Rapley T. Transitional care in clinical networks for young people with juvenile idiopathic arthritis: current situation and challenges. Clin Rheumatol [Internet]. 2015/04/30. abril de 2016;35(4):893-9. Disponible en: https://pubmed.ncbi.nlm.nih.gov/25920453

46. Green Corkins K, Miller MA, Whitworth JR, McGinnis C. Graduation Day: Healthcare Transition From Pediatric to Adult. Nutr Clin Pract Off Publ Am Soc Parenter Enter Nutr. febrero de 2018;33(1):81-9. https://doi.org/10.1002/ncp.10050

47. Gold A, Martin K, Breckbill K, Avitzur Y, Kaufman M. Transition to adult care in pediatric solid-organ transplant: development of a practice guideline. Prog Transplant. junio de 2015;25(2):131-8. DOI: 10.7182/pit2015833

48. Kreuzer M, Prüfe J, Tönshoff B, Pape L. Survey on Management of Transition and Transfer From Pediatric- to Adult-based Care in Pediatric Kidney Transplant Recipients in Europe. Transplant direct [Internet]. el 1 de junio de 2018;4(7):e361-e361. Disponible en: https://pubmed.ncbi.nlm.nih.gov/30046651

49. Tilton AH, de Gusmao CM. Transition From Pediatric to Adult Neurologic Care. Continuum (Minneap Minn). febrero de 2018;24(1, Child Neurology):276-87. DOI: 10.1212/CON.0000000000000570

50. Volkmar FR, Jackson SLJ, Hart L. Transition Issues and Challenges for Youth with Autism Spectrum Disorders. Pediatr Ann. junio de 2017;46(6):e219-23. DOI: 10.3928/19382359-20170519-03 